\title{
By any other name: consensus in the nomenclature of perioperative neurocognitive disorders
}

\author{
Hilary P. Grocott, MD, FRCPC, FASE • Gregory L. Bryson, MD, FRCPC
}

Received: 24 July 2018/Accepted: 24 July 2018/Published online: 16 October 2018

(c) Canadian Anesthesiologists' Society 2018

In this issue of the Journal, a consensus document from Evered et al. describes a new nomenclature paradigm for understanding perioperative delirium and cognitive disorders. ${ }^{1}$ This important document serves to establish uniformity in this growing and continually evolving area of research where considerable heterogeneity in defining these disorders has previously existed. Indeed, this heterogeneity has been a significant hindrance to progress in better understanding this increasingly recognized group of neurologic disorders. Importantly, this article is also being simultaneously co-published in five other journals addressing the individual disciplines of anesthesia and Alzheimer's disease. Part of the reason for this wideranging co-publication is the importance of this topic to all of the journals that commonly publish in this area.

The nomenclature recommendations are highlighted by the alignment of perioperative neurocognitive disorders (NCD) with the Diagnostic and Statistical Manual for Mental Disorders, Fifth Edition (DSM-5). ${ }^{2}$ Readers familiar with this literature will note that delirium continues to be defined by the DSM-5. Common clinical delirium screening tools, such as the Confusion Assessment Method, simply operationalize the DSM-5 diagnostic criteria. ${ }^{3}$ The most notable change resulting from this alignment is the renaming of a condition that is

\section{H. P. Grocott, MD, FRCPC, FASE ( $\square)$}

Department of Anesthesiology, Perioperative \& Pain Medicine,

St. Boniface Hospital, University of Manitoba, CR3008 - 369

Tache Avenue, Winnipeg, MB R2H 2A6, Canada

e-mail: hgrocott@sbgh.mb.ca

\section{G. L. Bryson, MD, FRCPC}

Department of Anesthesiology \& Pain Medicine, Ottawa Hospital Research Institute, University of Ottawa, Ottawa, ON, Canada common in the anesthesia literature: postoperative cognitive dysfunction (POCD). Indeed, POCD is a research diagnosis dependent upon interim changes in neuropsychometric testing, and while clearly defined by the International Study of Postoperative Cognitive Dysfunction, ${ }^{4}$ POCD is unique to perioperative research. The terminology used in the DSM-5 is NCD. Similarly to POCD, the diagnosis of a NCD is based on neuropsychometric testing, but now adds the important constructs of cognitive concern and impairment of function. ${ }^{1}$

Cognitive concern is the report of the patient, caregiver, or clinician noting a subjective decline in cognitive function. This acknowledgement of the subjective sense that the patient's thinking has changed is common to many DSM-5 diagnoses and is now essential to the diagnosis of neurocognitive disorders following anesthesia and surgery. Neurocognitive disorders are now also further divided into minor and major categories based upon the severity of the deficit identified by neuropsychometric testing. Mild NCD is defined as a decline of one to two standard deviations in neuropsychometric test scores when compared with a normative control group. Major NCD requires a decline in testing scores of greater than two standard deviations and requires a documented impairment of the activities of daily living. Defined by Lawton and Brody in 1969, the instrumental activities of daily living include eight activities essential for independent living, including shopping, food preparation, and self-management of medication. ${ }^{5}$ Inability to perform these basic functions independently is a significant feature of many DSM-5 diagnoses and its inclusion here aligns perioperative and other conditions.

These new recommendations also acknowledge the complex perioperative environment where surgery, 
residual anesthetics, analgesics, and coexisting NCDs contribute to altered and fluctuating cognitive and functional assessment. Co-existent preoperative NCDs are also formally recognized as mild and major. Any NCDs noted within the first 30 days of surgery are classified as delayed neurocognitive recovery while those persisting from 30 days to 12 months are identified as "postoperative."

These new diagnostic terms, agreed upon by major anesthesia and Alzheimer's disease journals in the United Kingdom, United States, Australia, and Canada, should bring consistency among publications in defining these disorders. This has several advantages. Firstly, it reduces some of the uncertainty regarding what is actually being named. That is, we all need to speak the same language if we are going to make any headway in addressing this troublesome perioperative problem. Next, standardization of terminology will ensure consistency from one investigative group to another, a considerable advance in this particular field of research. Moving beyond anesthesia research, alignment of NCDs with DSM-5 permits clear and consistent communication of these conditions among specialists, such as surgeons, intensivists, internists, neurologists, and psychiatrists, with whom we share perioperative care. Furthermore, systematic reviews may be greatly assisted if there is commonality in defining the disorders being studied. It should also help shape future investigations by better understanding where the knowledge gaps exist and where progress needs to be made.

It is important to note that these new recommendations do not specify the specific neuropsychometric tests or testing batteries to be used in perioperative research. This is again consistent with DSM-5, which specifies only the criteria for diagnosis, not the means by which to assess them. Future work is required to standardize diagnostic tools used in perioperative cognitive research. The consistency of definition and focus on functional outcome in these new recommendations is, however, consistent with a broader movement within anesthesia research. The Journal has previously highlighted the importance of standardized outcomes that matter to patients in both research and clinical practice ${ }^{6,7}$ Evered and a number of the co-authors of these new recommendations are contributors to the COMPAC-StEP (Core Outcomes Measures in Perioperative and Anesthetic CareStandardized Endpoints in Perioperative Medicine) group and will standardize perioperative cognition and stroke research. $^{8}$

Though Shakespeare wrote that "a rose by any other name would smell as sweet", science and evidence-based practice demand clarity. Leading anesthesia journals, including the Canadian Journal of Anesthesia, have acknowledged these demands. We hope that the joint publication of these consensus recommendations will promote much needed clarity in the research and treatment of perioperative NCD.

\section{Quel que soit son nom : consensus sur la nomenclature des troubles neurocognitifs périopératoires}

Dans ce numéro du Journal, un document de consensus signé par Evered et coll. décrit un nouveau paradigme de nomenclature pour la compréhension du délirium et des troubles cognitifs périopératoires. ${ }^{1}$ Cet important document contribue à créer une homogénéité dans ce domaine de recherche grandissant et en évolution constante qui était jusque là caractérisé par l'hétérogénéité considérable concernant la définition de ces atteintes. De fait, cette hétérogénéité a constitué un obstacle significatif à une meilleure compréhension de ce groupe de troubles neurologiques de plus en plus reconnus. Cet article est également important dans la mesure où il est publié simultanément dans cinq autres journaux abordant les disciplines individuelles de l'anesthésie et de la maladie d'Alzheimer. La raison de cette co-publication à grande échelle est en partie l'importance de ce sujet pour tous les journaux qui publient habituellement dans ce domaine.

Les recommandations sur la nomenclature sont renforcées par l'harmonisation des troubles neurocognitifs (TNC) périopératoires avec le Manuel diagnostique et statistique des troubles mentaux, cinquième Édition (DSM-5). ${ }^{2}$ Les lecteurs connaissant ce document noteront que le délirium est toujours défini par le DSM-5. Les outils de dépistage courants du délirium clinique, telle que la Méthode d'évaluation de la confusion, rendent simplement opérationnels les critères diagnostiques du DSM-5. ${ }^{3}$ Le changement le plus important résultant de cette harmonisation est le nouveau nom donné à un trouble qui est courant dans les publications anesthésiques : le trouble cognitif postopératoire (TCPO/POCD postoperative cognitive dysfunction). Le TCPO est en fait un diagnostic de recherche qui dépend des changements intérimaires à des épreuves neuro-psychométriques et, bien qu'il ait été clairement défini dans l'étude internationale sur les troubles cognitifs postopératoires, ${ }^{4}$ le TCPO est spécifique à la recherche périopératoire. La terminologie utilisée dans le DSM-5 est «TNC ». Comme pour le TCPO, le diagnostic de TNC repose sur des épreuves 
neuro-psychométriques, mais il ajoute maintenant les domaines importants de préoccupation cognitive et d'altération de la fonction. ${ }^{1}$

La préoccupation cognitive est le signalement par le patient, l'aidant ou le clinicien d'un déclin subjectif des fonctions cognitives. Cette reconnaissance de l'impression que la pensée du patient s'est modifiée est commune à de nombreux diagnostics du DSM-5 et est maintenant essentielle au diagnostic de trouble neurocognitif après une anesthésie et une chirurgie. Les troubles neurocognitifs sont en outre scindés en catégories majeures et mineures en fonction de la sévérité du déficit identifié par l'épreuve neuropsychométrique. Un TNC léger est défini par un déclin d'un ou deux écarts-types aux scores des tests neuro-psychométriques par rapport à la norme d'un groupe témoin. Un TNC majeur requiert un déclin supérieur à deux écarts-types aux scores d'épreuves et une altération documentée d'activités de la vie quotidienne. Définies par Lawton et Brody en 1969, les activités instrumentales de la vie quotidienne incluent huit activités essentielles pour une vie indépendante, notamment le magasinage, la préparation des repas et la gestion autonome des médicaments. ${ }^{5}$ L'inaptitude à effectuer ces fonctions de base de façon autonome est une caractéristique significative de nombreux diagnostics du DSM-5 et son inclusion ici va dans le sens des troubles périopératoires et autres.

Ces nouvelles recommandations reconnaissent également l'environnement périopératoire complexe dans lequel la chirurgie, les agents anesthésiques résiduels, les médicaments analgésiques et les TNC coexistants contribuent à une évaluation cognitive et fonctionnelle altérée et fluctuante. Les TNC préopératoires coexistants sont aussi formellement reconnus comme légers et majeurs. Tout TNC noté au cours des 30 premiers jours suivant l'opération est classé comme étant une récupération neurocognitive retardée, tandis que ceux persistant entre 30 jours et 12 mois sont qualifiés de «postopératoires ».

Cette nouvelle terminologie diagnostique, acceptée d'un commun accord par les principaux journaux d'anesthésie et sur la maladie d'Alzheimer au Royaume-Uni, aux États-Unis, en Australie et au Canada devrait apporter une cohérence parmi les publications dans la définition de ces troubles. Cela a plusieurs avantages. Tout d'abord, cela réduit une partie de l'incertitude concernant ce qui est vraiment nommé. C'est-à-dire que nous devons tous parler le même langage si nous voulons progresser dans la résolution de cette problématique trouble périopératoire. Ensuite, la normalisation de la terminologie assurera une cohérence entre les groupes de chercheurs, un progrès considérable dans ce champ de recherche particulier.
Dépassant la recherche en anesthésie, l'harmonisation des TNC avec le DSM-5 permet une communication claire et homogène sur ces troubles entre les spécialistes, tels que les chirurgiens, intensivistes, neurologues et psychiatres avec lesquels nous partageons les soins périopératoires. En outre, les études systématiques bénéficieront largement d'une définition commune des troubles étudiés. Cela devrait aussi contribuer à de futures recherches, en permettant de mieux comprendre où sont les lacunes dans les connaissances et où il $\mathrm{y}$ a lieu de faire des progrès.

Il est important de noter que ces nouvelles recommandations ne précisent pas quelles épreuves neuro-psychométriques ou batteries de tests doivent être utilisées dans la recherche périopératoire. Cela est aussi cohérent avec le DSM-5 qui indique uniquement les critères diagnostiques et non les moyens pour y parvenir. Du travail sera encore nécessaire pour normaliser les outils diagnostiques utilisés dans la recherche cognitive périopératoire. L'homogénéité de la définition et l'importance accordée au résultat fonctionnel de ces nouvelles recommandations correspondent toutefois à un plus vaste mouvement au sein de la recherche en anesthésie. Le Journal a déjà souligné l'importance de la normalisation des critères de résultats qui ont de l'importance pour les patients, à la fois au cours des recherches et en pratique clinique. ${ }^{6,7}$ Evered et plusieurs co-auteurs de ces nouvelles recommandations contribuent au groupe COMPAC-StEP (sur les principales mesures des résultats dans les critères d'évaluation normalisés des soins anesthésiques et périopératoires en médecine périopératoire); ils normaliseront aussi la recherche sur la cognition périopératoire et les AVC. ${ }^{8}$

Bien que Shakespeare ait écrit que «ce que nous appelons une rose embaumerait autant sous un autre nom », la science et la pratique reposant sur les données probantes ont besoin de clarté. Les principaux journaux d'anesthésie, dont le Journal canadien d'anesthésie, ont reconnu ces demandes. Nous espérons que la publication conjointe de ces recommandations de consensus encouragera cette clarté grandement nécessaire pour la recherche et le traitement des TNC périopératoires.

Conflicts of interest None declared.

Editorial responsibility This submission was handled by Dr. Philip M. Jones, Associate Editor, Canadian Journal of Anesthesia.

Conflits d'intérêts Aucun déclaré.

Responsabilité éditoriale Cet article a été traité par le Dr Philip M. Jones, rédacteur adjoint, Journal canadien d'anesthésie. 


\section{References}

1. Evered L, Silbert B, Knopman DS, et al. Recommendations for the nomenclature of cognitive change associated with anaesthesia and surgery - 2018. Can J Anesth 2018. DOI: https://doi.org/10.1007/ s12630-018-1216-x.

2. American Psychiatric Association. Diagnostic and Statistical Manual of Mental Disorders. 5th ed. Arlington, VA, USA: American Psychiatric Publishing; 2013.

3. Inouye SK, van Dyck CH, Alessi CA, Balkin S, Siegal AP, Horwitz RI. Clarifying confusion: the confusion assessment method. A new method for detection of delirium. Ann Intern Med 1990; 113: 941-8.

4. Rasmussen LS, Larsen $K$, Houx $P$, et al. The assessment of postoperative cognitive function. Acta Anaesthesiol Scand 2001; 45: $275-89$
5. Lawton MP, Brody EM. Assessment of older people: selfmaintaining and instrumental activities of daily living. Gerontologist 1969; 9: 179-86.

6. Boney O, Moonesinghe SR, Myles PS, Grocott MP. Standardizing endpoints in perioperative research. Can J Anesth 2016; 63: 15968.

7. Myles PS. Perioperative outcomes: Are we asking the right questions? Can J Anesth 2016; 63: 138-41.

8. Myles PS, Boney $O$, Botti $M$, et al. Systematic review and consensus definitions for the Standardised Endpoints in Perioperative Medicine (StEP) initiative: patient comfort. Br J Anaesth 2018; 120: 705-11. 